\title{
Modelling of the Ward Round Process in a Healthcare Unit
}

\author{
Adid Ali Fareedi and Vladimir Tarasov \\ School of Engineering, Jönköping University, \\ P.O. Box 1026, 55111 Jönköping, Sweden \\ alab08af@student.hj.se, vladimir.tarasov@jth.hj.se
}

\begin{abstract}
Information systems (IS) are nowadays extensively used to support all kinds of activities in healthcare organisations. Enterprise modelling can help to make the use of IS in healthcare more effective by providing process and domain models reflecting a particular healthcare unit. This paper proposes a model of the ward round process in a healthcare unit. The proposed model identifies the roles of medical professionals, tasks that can be performed according to the personnel's competences, and activities that are carried out as part of the tasks to achieve goals of the ward round process. A formal approach has been used to implement the modelling results in the form of an ontology. Such formal ontologies can support improvement and development of IS in healthcare. We learned that modelling workshops are important for development of models that can be formalized in a machine-readable form.
\end{abstract}

Keywords: Enterprise modelling, conceptual modelling, ontologies, healthcare process, ward round.

\section{Introduction}

Information systems (IS) are nowadays part of almost every activity or process in healthcare organisations. IS are intended to support the medical personnel and make their work more efficient. Yet, the use of IS in healthcare needs to be improved [1]. Current IS do not always meet the needs of healthcare professionals and patients. To achieve better use of IS, we need to understand work processes, which are to be supported by IS, the needs of the people who are going to use IS, and the details of the healthcare domain. Enterprise modelling provides means to model the current situation and to describe work processes, tasks, roles, and resources. Thus, enterprise modelling can help to make the use of IS in healthcare more effective by providing models reflecting a particular healthcare unit. Conceptual modelling is an important part of enterprise modelling [2]. The advantage of conceptual modelling is that the resulting model can be formalised as an ontology in a machine-readable form, that can be directly used to improve existing IS or build new ones. Ontological modelling methods have been used to support development of healthcare IS in may cases (e.g. 3,45]).

The purpose of this paper is to propose a model of the ward round process at Ryhov hospital in Jönköping. The model is implemented as a formal ontology. 
Existing IS have not been able to provide full support to medical professionals in all different contexts of the healthcare processes at Ryhov hospital. Development of a formal model of the ward round process is needed to help to customize current IS to improve information flow, i.e. to provide the required information to an individual according to his/her role and competence in a specific context of the ward round at the Ryhov Hospital. The main contribution of the work is that it presents a detailed representation of the ward round that can be used for development or improvement of healthcare IS. Such IS can provide better support for the healthcare professionals to improve the quality of the patient's treatment. The ontology-based implementation of the model can be also directly utilised in IS to improve information flow in a healthcare organisation.

The paper is structured as follows. Section 2 briefly describes modelling in healthcare, explains the Ward Round process and presents the case. The method used for carrying out this study is presented in section 3 Section 4 details the developed model of the Ward Round process from the model design to the implementation. Section 5 discusses the lessons learned during the model development. The summary of the results and future work are presented in section 6 .

\section{Background}

This section provides short overview of different modelling approaches applied in the healthcare area as well as briefly describes the ward round and its importance in the medical institutions. The section ends with an introduction of the modelling case.

\subsection{Modelling in Healthcare}

Enterprise modelling (EM) plays an important role in the healthcare sector because it promises to improve the use of healthcare information systems (IS). EM can be used to analyse patterns of healthcare activities, ensure fulfilment of the end-user's needs and requirements, build a systematic view of patient-centred processes, and help healthcare institutions to improve internal knowledge and understanding. EM also contributes to supporting IS that are intended to help healthcare professionals to manage patient treatment activities and document all the events of the care processes [6]. Enterprise modelling approach has been used for supporting a set of structured, goal/problem-driven models for capturing, structuring and representing organizational knowledge, and designing different perspectives of process modelling: functional, informational, organizational and behavioural 789 .

New business demands imposed on enterprises and organisations require EM methods that are more formal, i.e. conceptual modelling methods [2]. Ontological modelling is the key tool in formal conceptual modelling since "an ontology is an explicit specification of a conceptualization" [10. Ontology development is a method to formalize conceptual models in machine-readable form that can be utilized for improvement of existing healthcare IS or development of new IS. 
Nowadays, the importance of ontological modelling has been acknowledged due to its usage in the healthcare sector. Ontological modelling helps to acquire knowledge in the machine-understandable form, which results in making IS more intelligent. This approach has been used in the healthcare area for modelling of the patient's treatment process and for improvement of the quality of healthcare services [3. To achieve the latter, efficient healthcare workflow management systems are needed that support data exchange between different processes and provide relevant information timely, effectively and according to the demand of individuals. An ontological knowledge framework presented in 3 covers the hospital processes from patient records to hospital resources and administrative tasks. An adaptive workflow system constructs a process and resource model, which include different levels to represent the patient treatment processes: patient's admission, treatment planning, and discharge.

Different approaches are utilized for ontological modelling in the healthcare sector nowadays. The archetype pattern approach is employed to develop an ontology for a healthcare IS and to provide shared understanding of the healthcare processes 4]. The ontology-driven multi-agent approach provides a framework to help the medical professionals to interact and collaborate effectively [5]. The competence management approach is used to develop competence of healthcare personnel based on goals in order to enhance competence at the enterprise level. The case study of Municipal Hospital of Karlsruhe details this competence management approach [1].

\subsection{The Ward Round Process}

The ward round is the process intended for making decisions concerning the patient's treatment process by medical professionals and resource personnel [12]. It provides opportunity for multidisciplinary teams to carry out different activities: patient's examination, treatment and discharge from the hospital [13]. Ward rounds support patient treatment planning, prognosis formulation and analysis of social, psychological, rehabilitation and placement issues [12]. Learning opportunities are supported by ward round as well [14]. Ward round is categorized into traditional and modern ones. The traditional ward round is authoritatively led by senior hospital personnel who have responsibility for making decisions in the patient treatment process. On the contrary, the modern ward round relies on expertise of both senior and junior colleagues and aims at achieving consensus in order to improve the quality of healthcare from the patient perspective [12 15].

\subsection{The Ryhov Hospital Case}

The work described in this paper was partly carried out within the project "Bridging the Gaps". It is a research project aimed at improving healthcare and driven by The Jönköping Academy for Improvement of Health and Welfare1.

${ }^{1}$ http://www . jonkopingacademy.se 
The healthcare processes modelled in Sect. 4 represent the ward round at Ryhov Hospital in Jönköping, Sweden.

During the ward round, healthcare professionals with different competences, roles and skills gather at the appointed time to assess the patient's status, collect information for making diagnosis as well as plan the patient's treatment process. The head nurse consults the EBBA information system to get data about availability of vacant beds to enhance the treatment pace of normal patients, emergency patients, patients sent from other healthcare units, and patients admitted through the certain ward. Every morning the medical staff receive information regarding a particular patient in the form of medical reports from different information channels like Cambio Cosmic (electronic medical record system), EBBA (patient-admittance status system), and a database connected to the pathology laboratory. These systems are integrated and share information in real-time manner to support intelligent decision making during the ward round in a healthcare unit. The details about the case can be found in [15].

\section{$3 \quad$ Method}

Modelling of the ward round case (see Sect. 2.3) has been partly carried out as a collaborative activity with participants of different backgrounds such as modelling, medical, and IT ones. As soon as the goal was to create a healthcare process model as a formal ontology, the other part of the modelling was only performed by the modelling experts because it required very specialized expertise. The formal ontology was the intention since it could be used to support development and improvement of healthcare IS. During the modelling activity we followed a number of steps [15]:

- Arrange a modelling workshop with domain and modelling experts,

- Acquire knowledge about the ward round during the modelling workshop,

- Prepare the case data based on the modelling workshop,

- Support the case with the study of related literature,

- Analyse the results of the domain modelling and literature review,

- Determine appropriate tasks, processes, activities, and roles comprising the ward round,

- Describe steps needed to perform the tasks,

- Select a suitable methodology for implementation of the model as a formal ontology and perform the implementation,

- Verify and evaluate the constructed model through domain experts' assessment and description logic queries.

The modelling workshops have been used as one of the main techniques in this study. We have conducted two workshops. The first one was intended to acquire relevant knowledge from the domain experts for creation of the model of the ward round at Ryhov hospital in Jönköping. In this workshop, we have engaged multidisciplinary professionals such as modelling experts, medical practitioners and IT experts with their experiences, observations and different expertise to 
develop a model of the ward round. Before presenting the results in the second modelling workshop, we developed a formal model that can be useful for the improvement of healthcare IS. During the second workshop we presented the results of the modelling to the domain experts to ensure the quality of the model. We also discussed design issues like individual roles and key responsibilities in the ward round, and different resources that were used in ward rounds.

\section{Model of the Ward Round Process}

This section presents design and briefs implementation of the model of the ward round and explains different activities which are necessary to perform the ward round process. The details of the model are described in [15]. Before considering the ward round for a patient's treatment, it is important to evaluate the patient's condition at the time of admission. The criteria for the admission procedure are also described in [15].

\subsection{Design of the Model}

The design of the model, which resulted from the first modelling workshop, is depicted in Fig. 1. It shows the structure of the ward round at Ryhov hospital in Jönköping. The ward round is the centre of activities where different hospital professionals gather for treatment of the patient. The overall ward round process is divided into three sub-processes, which consequently support each other. These sub-processes are the pre-ward round process, the ward round process and postward round process.

The ward round process includes different tasks that are carried out to treat the patient's illness. Hospital professionals have different roles in the process and the ward round has different goals to achieve quality in the patient's treatment process. These goals lead to different tasks, which include activities in the patient's treatment process. Different resources are utilized for the completion of each task. Resources can be patient treatment documents, medical equipment, IS, competence resources and database systems.

\subsection{Ward Round Process}

In ward round process modelling, we have divided medical professionals into three designated teams: designated team 1, designated team 2 and designated team 3. These teams consist of multidisciplinary professionals who are responsible for initiation of the sub-processes: the pre-ward round process, ward round process, and post-ward round process. The teams perform different tasks and activities and use resources to achieve the set goals. Designated team 1 includes a junior practitioner, head nurse, additional nurse, occupational therapist, and laboratory personnel that are responsible for update of the patient's medical record in the pre-ward round process. Designated team 2 consists of a consultant, senior and junior practitioners, nurse, and occupational therapist to perform the 


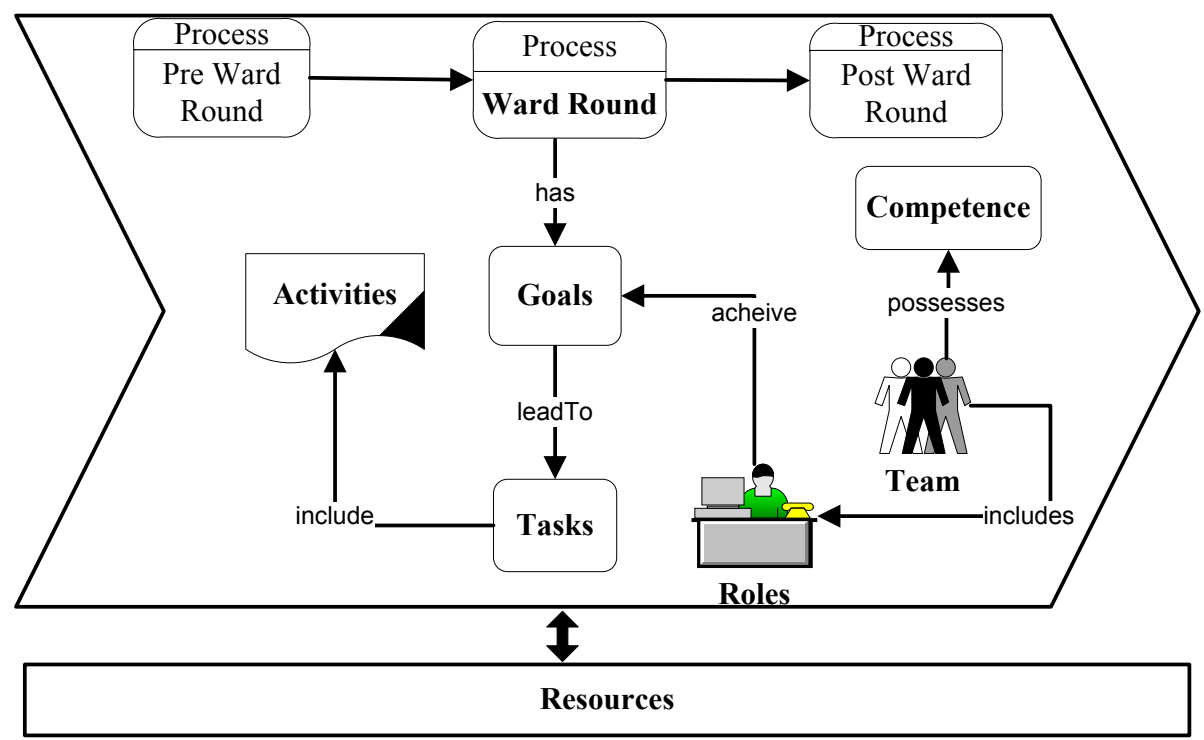

Fig. 1. Overview of the model

ward round process for planning of the patient's treatment. Designated team 3 is also comprised of senior and junior practitioners, an occupational therapist and medical student for group discussion and evaluation of the current analysis of the patient's illness in the post-ward round process.

We have chosen designated team 2 for a more detailed description. This team initiates the ward round process with the goal of timely result receipt, the task of patient treatment planning, and the activity of identifying medical problems in the patient's treatment in the ward round session. Designated team 2 utilizes the resources EBBA and Cosmic to achieve quality of patient treatment in the ward round process. The process of modelling included identification of goals, tasks, activities, roles, and resources, which are listed in the following subsections.

Goals. The following goals are important to achieve quality of the patient treatment planning process in the ward round:

- To analyse the actual causes of the patient's illness,

- To support quality management of planning in the ward round,

- To prepare a pool of additional questions related to the patient's previous life or medical, psychiatric, sexual, family and social aspects,

- To achieve effective decision making in immediate or long-term treatment planning to tackle the patient's illness.

Tasks. The tasks of planning of the patient's treatment. 
Activities. The activities that help to carry out the above mentioned task are:

- To consider a differential diagnosis in the patient's treatment planning in the ward round process [16],

- To formulate a differential diagnosis, which involves review of the patient's history and clinical examination of the findings in the patient's treatment planning [16],

- To identify medical problems connected with the patient's illness and to construct a management plan to tackle the disease [16,

- To ask additional questions to clarify different aspects of the patient's history regarding medicine, effects of medicine, sensitive aspects of psychiatric and sexual nature, and evaluate risk factors related to consequences of the illness, family history and social history [16],

- To decide on the patient's treatment plan according to the patient's illnesseither an immediate plan or long-term one [16].

Roles. The following roles are extracted from the case during the modelling of the ward round process:

- A consultant is responsible for identification of all possible causes after the review the patient's history during patient treatment planning,

- A consultant is also responsible for identification of medical problems related to the patient's illness during patient treatment planning,

- A senior practitioner formulates a differential diagnosis after the review of the patient's history and examination of the clinical findings,

- A junior practitioners assists a senior one in collection of the examination findings,

- A nurse provides medical reports related to the patient or the patient's portfolio,

- A junior practitioners is also responsible for asking the patient additional questions to support the quality of patient treatment planning,

- A senior practitioner decides on the treatment plan according to the patient's illness - either an immediate plan or long-term plan.

Resources. To perform the activities of the task of patient treatment planning, several resources are utilized during the ward round in Ryhov hospital at Jönköping:

- The information systems Cosmic and EBBA are used for acquiring information related to the patient in the process of patient treatment planning,

- The laboratory database system is used to send reports on the patient medical tests to nurses for patient treatment planning,

- Patient medical reports are used for the assessment of the patient treatment process. 


\subsection{A Process View of the Ward Round}

We have employed EKD modelling technique (see [17]) to design the ward round model shown in Fig. 1. This sections describes key processes, external processes and information sets, which are depicted in Fig. 2. They constitute the process view of the ward round model. An external process is a collection of activities that are located outside the scope of the organizational activity area and communicate with processes or activities of the problem domain area 17. An information set is a set of information, which is sent from one process to another one to facilitate information flow between the processes [17. The following processes represent the working flow of the ward round:

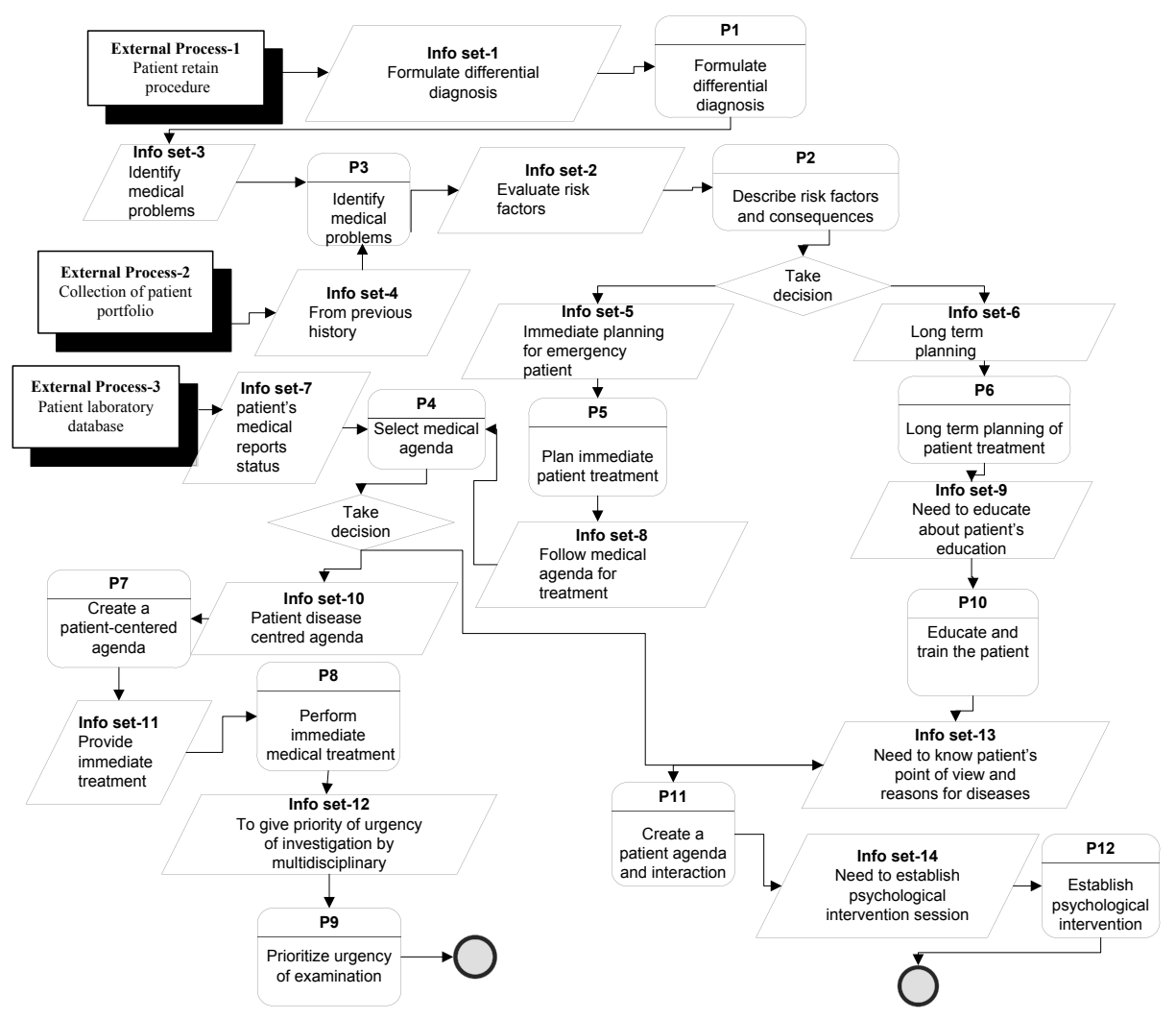

Fig. 2. The process perspective in the ward round model

1. Formulate differential diagnosis. This process helps to describe a number of steps to determine the problem at the time of diagnosis.

2. Describe risk factors and consequences. This process explains risk factors.

3. Identify medical problems. It details medical problems in the diagnosis. 
4. Select medical agenda. This process chooses a medical treatment agenda: a long-term one or short-term one.

5. Plan immediate patient treatment. This describes emergent patient treatment in the ward round.

6. Long term planning of patient treatment. Long term treatment is described for a normal patient in the ward round.

7. Create a patient-centred agenda. This process determines particular disease treatment.

8. Perform immediate medical treatment. Immediate treatment is provided to the patient in the ward round.

9. Prioritize urgency of examination. It establishes what data are most necessary to conduct the ward round.

10. Educate and train the patient. One needs to know the patient's point of view and explain reasons for disease treatment in the ward round.

11. Create a patient agenda and interaction. This process establishes good interaction with the patient in the ward round.

12. Establish psychological intervention. A psychological intervention session needs to be established during the ward round.

\subsection{Implementation of the Model}

This section briefly explains the implementation of the ward round model (detailed description can be found in [15]). A formal approach has been used which resulted in an ontology. We have used the Web Ontology Language (OWL) 2 and the Protégé ontology editor 3 to develop the ontology-based implementation. The constructed ontology contains 146 classes, 76 object properties, 1 data property, and 122 individuals. Fig. 3 depicts an overview of the ontology that contains different entities involved in the ward round and relations between them.

The medical staff members are involved in different processes (pre-ward round, ward round, and post-ward round) according to their competences and assigned roles. The assignment of the roles of a consultant or senior practitioner determines which process is initiated. The selection of a process is followed by the medical agenda [18] in the patient treatment procedure. Different processes in the ward round model utilize diverse resources for receiving related information from the information channels according to the rules set by a healthcare unit. These processes have particular goals. Every goal leads to a number of tasks that include activities. "Component selection criteria" is a component that supports the selection of the right person to perform his/her responsibilities according to the assigned roles. The selection is carried out based on the person's competences and skills.

To illustrate the use of the constructed ontology for modelling of a real situation from the ward round, we give an example scenario describing the role of a junior practitioner and show its ontological representation in Fig. 4.

\footnotetext{
2 http://www.w3.org/TR/owl2-overview

3 http://protege.stanford.edu
} 


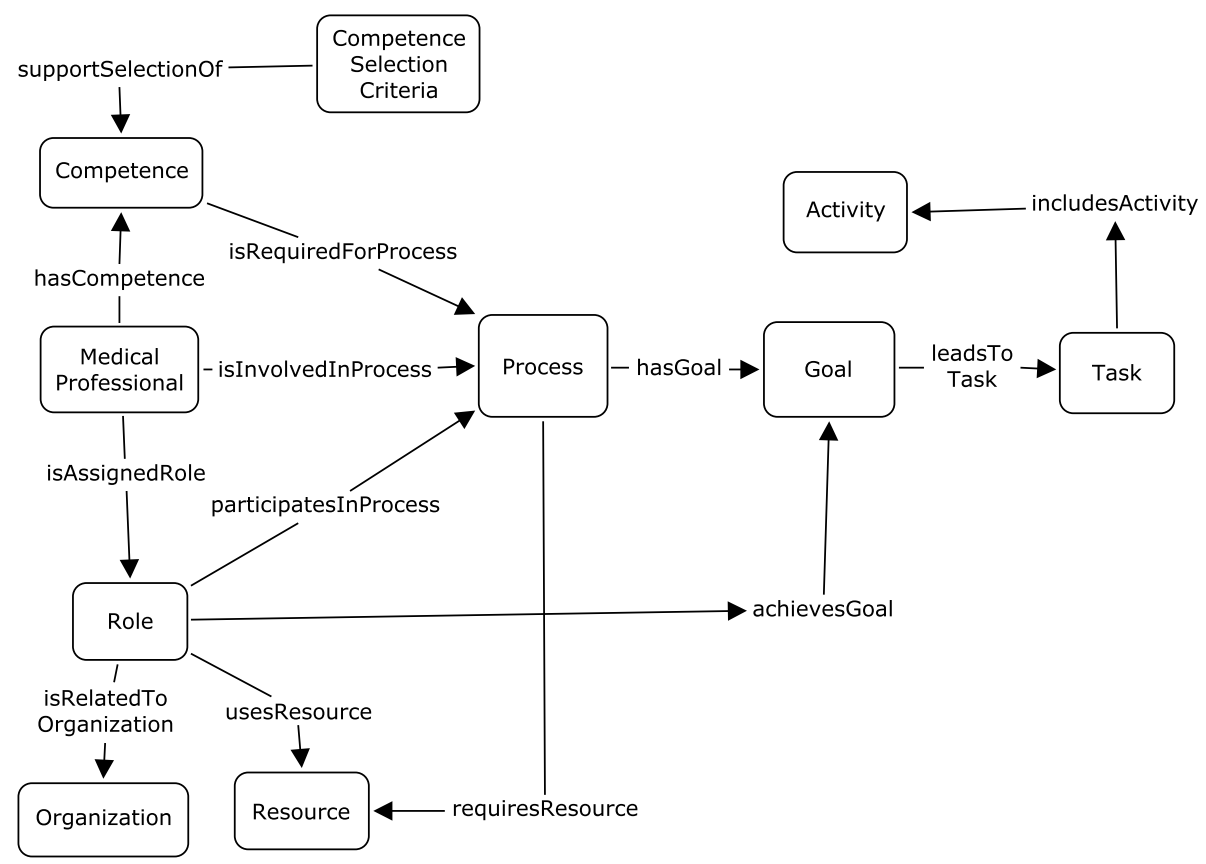

Fig. 3. Overview of the ontology-based implementation of the ward round model

Scenario. The junior practitioner role is assigned to Person A who works at Ryhov Hospital in Jönköping and belongs to designated team 2. Person A participates in the ward round process and performs different tasks like patient treatment planning. Person A carries out several activities such as to complete the physical examination to determine medical problems for patient treatment planning. Person A's main responsibilities are to visit a particular ward daily and check the patient's status from the Cosmic information system to inform the senior staff. Person A has several competences: cultural, occupational and general ones (they are not shown for breviety).

\subsection{Evaluation of the Model}

The goal of the evaluation was to achieve quality of the work and get assurance that the model represents the ward round in the correct way. The evaluation of the model was done in two ways [15]:

1. Using description logic (DL) queries. We have used the DL Query Tab in Protégé 4 to verify competency questions. They help to confirm that the ontology has enough information to answer these questions, which are related 


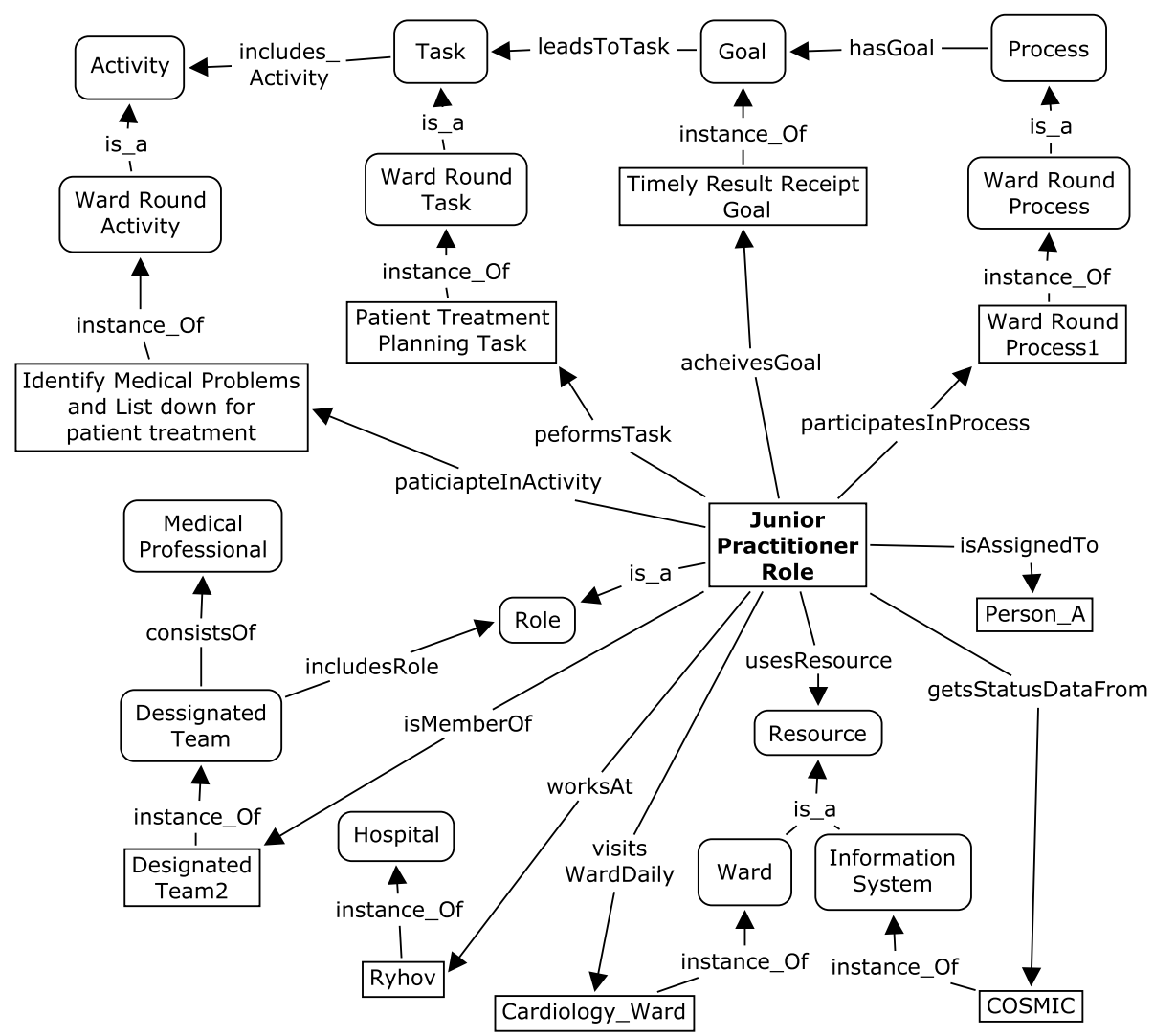

Fig. 4. The junior practitioner role represented with the constructed ontology

to the domain (the ward round). Table 1 shows an example of one competency question with a DL query and Fig. 5 presents the results of executing the DL query in the Protégé editor.

2. $2^{\text {nd }}$ modelling workshop. During this workshop, we have presented holistic view of the model design that illustrates how medical professionals with different roles and competences initiate different processes and perform different activities to achieve the ward round goals. We have also exemplified the model of the Ryhov hospital case with a simple scenario that shows representation of a practical situation. These formal modelling results reflect detailed representation of the ward round, which can be used in healthcare IS to improve information flow in the ward round context. Thus, during the $2^{\text {nd }}$ modelling workshop we have presented the final modelling results to the domain experts to verify the model, get feedback and suggestions for future improvement. 
Table 1. An example of a competency question and a DL query

\begin{tabular}{lll}
\hline Competency question & \multicolumn{1}{c}{ DL query } & \multicolumn{1}{c}{ Query results } \\
\hline Who are the members & Role and & Medical Individuals: \\
involved in ward round & initiateProcess & OccupationalTherapist, \\
team? & value & AdditionalNurse, SeniorPractitioner, \\
& WardroundProcess1 & HeadNurse, JuniorPractitioner \\
\hline
\end{tabular}

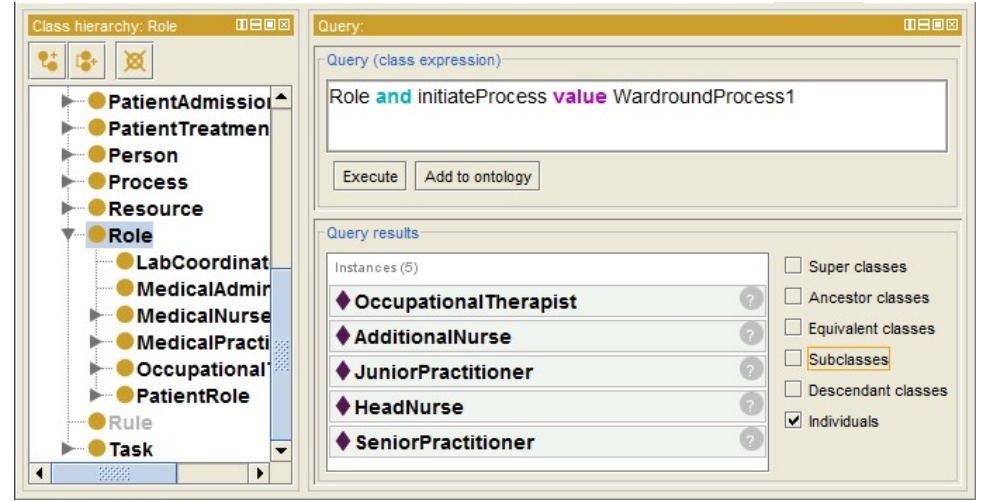

Fig. 5. The results of executing the DL query in the DL query tab in Protégé

\section{Lessons Learned}

The main objective of the modelling workshops was to develop a model that can be formalised as an ontology in a machine-readable form to support improvement and development of IS in healthcare. We learned that using modelling workshops was very productive to acquire relevant knowledge about the Ward Round to develop an ontological model. By using modelling workshops and other modelling techniques like EKD [17], we were able to develop a model including tacit knowledge acquired from the domain experts and then implement the model in a form that allows for improvement of IS use in different domains like healthcare. Meanwhile the formal part of the modelling was necessary to carry out without involvement of the domain experts as soon as formal techniques, which require very special expertise, would confuse the domain experts.

The steps, which we described in Sect. 3, are well suited to develop any model in the healthcare sector in the systematic way. However, we have observed that participation of only multidisciplinary professionals with different expertise like modelling or use of IT in healthcare during the modelling workshops is not enough. We should also invite healthcare professionals to obtain better knowledge of intrinsic details from the healthcare domain. This may help to develop better ontological models in healthcare. 
It is also important to include healthcare professionals in the modelling workshops from the beginning because it is quite helpful for modellers to learn and get experience from different domain professionals and to train to work with participants with mixed culture and background. This work pattern helps the modellers to present the modelling results to the domain experts because they already familiar with the experts. It also creates synergy effects among the participants and improves the quality of the work. According to our experience, we can conclude that modelling workshops are a good opportunity specifically for new-comers in modelling. Novice modellers can quickly learn how to perceive the domain and choose a suitable methodology for modelling from the other multidisciplinary participants.

We have arranged two modelling workshops in our research work but we think that it is not sufficient. During the $1^{\text {st }}$ workshop, we familiarised ourselves with the participants and got "know-how" about the domain. In the $2^{\text {nd }}$ workshop, we presented the results in the form of models to the domain experts to get feedback. We suggest to conduct more workshops with healthcare professionals for improvement and assessment of the model.

We have presented our results to the domain experts during the modelling workshops in diagrammatic form that provided overview of the ward round model in the holistic way. This approach facilitates broad understanding of how the objects in the domain are connected with each other and what the information flow is. We have also used tabular form to present our results to the domain experts. The tabular form allowed them to get additional details about the elements in the ontology-based model. We have learned that both forms are useful for presentation of modelling results.

Different modelling tools have been considered, especially data and software modelling tools. However, finally we chose specialized tools for ontology-based model construction. We think that ontology editors like Protégé 44 and Top Braid Composer 5 are appropriate for model construction. During the development we have found that these tools are good because they allow for both expressiveness in models and evaluation of the modelling results.

\section{Conclusions}

This paper proposed a model representing the ward round process at Ryhov hospital in Jönköping. The model contains individual roles to be taken on by medical professionals while carrying out tasks to achieve effective patient treatment in the ward round process. During this process multidisciplinary professionals form different teams to perform tasks to achieve goals of the ward round. The model also shows different processes at different stages of the ward round. The hospital personnel have roles according to their competences required to perform activities in these processes.

\footnotetext{
4 http://protege.stanford.edu

5 http://www.topquadrant.com/products/TB_Composer.html
} 
The work was carried out based on two modelling workshops and literature study. We have implemented the constructed model in the form of a formal ontology. The ontological implementation has been developed using the Web Ontology Language (OWL) 6 and the Protég $\sqrt{7}$ ontology editor. For the evaluation of the results, we have utilized two ways: description logic (DL) queries and a $2^{\text {nd }}$ modelling workshop to verify the constructed model.

This work suggests that formal modelling techniques are important for the healthcare sector. The constructed ontology-based implementation of the model can be used to represent information needs of the healthcare professionals in the ward round patient treatment process in a healthcare unit. The formal ontology can be utilised directly in IS, which allows for the use of semantic techniques to improve information flow in the ward round process. For example, the ontology can be employed to improve the Cambio Cosmic IS, which is used at Ryhov hospital, in order to provide the doctors and nurses with the needed patient records, lab test results and so on according to their way of working during the ward round.

During the work we have learned that more modelling workshops are needed to create models that represent the domain in a more detailed and accurate way. Healthcare professionals are encouraged to be included in the modelling workshops from the very beginning. Diagrammatic and tabular forms are appropriate to communicate results of formal modelling methods to the healthcare professionals.

Our first priority for future work is to further validate the constructed model by testing it in IS in order to provide better healthcare services. We will also continue development of formal conceptual models in healthcare that can support improvement of information flow and development of better healthcare IS. The same modelling technique can be used for construction of ontological models in other areas like inter-professional interaction during the ward round process and doctorpatient communication, unmet patient needs after the ward round session, patient and student perspectives of teaching optimization, role and place of relatives, consultant vs. junior doctor rounds, quality of notes and patient communication, and alternatives to the bed-to-bed round [12. These areas were out of the scope of this paper but they can be used for further research in healthcare modelling.

Acknowledgements. The authors would like to thank Dr Klas Gäre and MSc Eva Lindholm for their active participation in the modelling workshops and valuable contribution to the discussion of the model.

Some parts of the presented research were carried out within the project "Bridging the Gaps" supported by the Vinvård research program, which is financed by VINNOVA and the Vardal Foundation in coordination with the Swedish Association of Local Authorities and Regions.

\section{References}

1. Littlejohns, P., Wyatt, J.C., Garvican, L.: Evaluating computerised health information systems: Hard lessons still to be learnt. BMJ 326(7394), 860-863 (2003)

\footnotetext{
6 http://www.w3.org/TR/ow12-overview

7 http://protege.stanford.edu
} 
2. Loucopoulos, P., Kavakli, V.: Enterprise knowledge management and conceptual modelling. In: Goos, G., Hartmanis, J., van Leeuwen, J., Chen, P., Akoka, J., Kangassalu, H., Thalheim, B. (eds.) Conceptual Modeling. LNCS, vol. 1565, pp. 123-143. Springer, Heidelberg (1999)

3. Dang, J., Hedayati, A., Hampel, K., Toklu, C.: An ontological knowledge framework for adaptive medical workflow. Journal of Biomedical Informatics 41(5), 829-836 (2008)

4. Raghupathi, W., Umar, A.: An archetype pattern driven approach to ontology development in health care information systems. In: Kendall, J.E. (ed.) Proceedings of the 39th Annual Meeting of the Decision Sciences Institute, pp. 4151-4156. Decision Sciences Institute, Atlanta (2008)

5. Ganendran, G., Tran, Q.N., Ganguly, P., Ray, P., Low, G.: An ontology-driven multi-agent approach for healthcare. In: Webb, R., Ribbons, R.M., Dall, V. (eds.) HIC 2002: Proceedings: Improving Quality by Lowering Barriers, pp. 464-469. Brunswick East, Vic., Health Informatics Society of Australia (2002)

6. Staccini, P., Joubert, M., Quaranta, J.F., Fieschi, D., Fieschi, M.: Modelling health care processes for eliciting user requirements: A way to link a quality paradigm and clinical information system design. International Journal of Medical Informatics 64(2-3), 129-142 (2001)

7. Shahzad, K., Zdravkovic, J.: A goal-oriented approach for business process improvement using process warehouse data. In: Aalst, W., Mylopoulos, J., Rosemann, M., Shaw, M.J., Szyperski, C., Persson, A., Stirna, J. (eds.) PoEM 2009. LNBIP, vol. 39, pp. 84-98. Springer, Heidelberg (2009)

8. Persson, A., Stirna, J., Aggestam, L.: How to disseminate professional knowledge in healthcare: The case of skaraborg hospital. Journal of Cases on Information Technology (JCIT) 10(4), 41-64 (2008)

9. Petersen, S.A., Bach, G., Svarlein, A.B.: Patient care across health care institutions: An enterprise modelling approach. In: Aalst, W., Mylopoulos, J., Rosemann, M., Shaw, M.J., Szyperski, C., Bommel, P., Hoppenbrouwers, S., Overbeek, S., Proper, E., Barjis, J. (eds.) PoEM 2010. LNBIP, vol. 68, pp. 91-105. Springer, Heidelberg (2010)

10. Gruber, T.R.: A translation approach to portable ontology specifications. Knowledge Acquisition 5(2), 199-220 (1993)

11. Kunzmann, C., Schmidt, A.: Ontology-based competence management for healthcare training planning: A case study. In: 6th International Conference on Knowledge Management, IKNOW 2006 (2006)

12. O'Hare, J.A.: Anatomy of the ward round. European Journal of Internal Medicine 19(5), 309-313 (2008)

13. Arasaratnam, R.: Medical ward rounds. Student BMJ 17, 98-99 (2009)

14. Stanley, P.: Structuring ward rounds for learning: Can opportunities be created? Medical Education 32(3), 239-243 (1998)

15. Abid, A.: Ontology-based model for the "ward-round" process in healthcare (OMWRP). Master's thesis, School of Engineering of Jönköping University (2010)

16. Cherry, J., Drury, A., Rigby, S.: Management plans. Student BMJ 16(10), 364-365 (2008)

17. Bubenko, Jr., J., Persson, A., Stirna, J.: D3: Appendix B. EKD User Guide. HyperKnowledge IST-2000-28401. Royal Institute of Technology (KTH), Stockholm, Sweden (2001)

18. Price, J., Leaver, L.: ABC of psychological medicine: Beginning treatment. BMJ 325(7354), 33-35 (2002) 